\title{
A Feasibility Study of Wearable Central Blood-Pressure Measurement System
}

\author{
Shuji Takahashi ${ }^{1}$, Yudai Komori ${ }^{2}$, Kyoji Nakajo ${ }^{1}$, Kazuhiro Motegi ${ }^{2}$, Yoichi Shiraishi ${ }^{2,}$, \\ Hiroshi Miyashita ${ }^{3}$
}

${ }^{1}$ Business Division, Cyber Physical System Department, AZAPA, Company, Limited, Naogya, Aichi, Japan

${ }^{2}$ Graduate School of Science and Technology, Gunma University, Ohta, Gunma, Japan

${ }^{3}$ Jichi Medical University Health Care Center, Jichi Medical University School of Medicine, Shimotsuke, Tochigi, Japan

\section{Email address:}

shuji-takahashi@azapa.co.jp (S. Takahashi),t12303009@gunma-u.ac.jp (Y. Komori), kyoji-nakajo@azapa.co.jp (K. Nakajo), motegi@gunma-u.ac.jp (K. Motegi), yoichi.siraisi@gunma-u.ac.jp (Y. Shiraishi),hrsm@jichi.ac.jp (H. Miyashita)

${ }^{*}$ Corresponding author

\section{To cite this article:}

Shuji Takahashi, Yudai Komori, Kyoji Nakajo, Kazuhiro Motegi, Yoichi Shiraishi, Hiroshi Miyashita. A Feasibility Study of Wearable Central Blood-Pressure Measurement System. Journal of Health and Environmental Research. Vol. 3, No. 4, 2017, pp. 62-71.

doi: $10.11648 /$ j.jher.20170304.12

Received: September 9, 2017; Accepted: October 11, 2017; Published: November 8, 2017

\begin{abstract}
A noninvasive estimation of central blood-pressure is required by the researchers or the medical practitioners in the cardiovascular treatment. In this study, first of all, a cardiovascular simulator based on the electrical circuits is described based on the model based approach and then, the representation of aorta model as a pressure transfer function is shown. This representation accelerates the simulator and then, makes it possible to estimate the central blood-pressure from a wearable gouge measurement in a real-time way. The system consisting of a main station, gateways and wearable blood-pressure gauges using one-board microcomputer is actually implemented. The functions and performances of the system are experimentally evaluated and the obtained results show the feasibility of central blood-pressure estimation and the localization of some examinees having wearable gauges with the practical accuracy and speed.
\end{abstract}

Keywords: Aorta Model, Cardiovascular Simulator, Central Blood-Pressure, IoT, Microprocessor, Transfer Function, Wearable Sensor

\section{Introduction}

The observation of dynamic blood flow in the cardiovascular system is very important whether it is in a normal state or in a diseased state. The vascular diseases are induced by atherosclerosis, arterial stiffening, aneurysm rupture and thromboembolism, etc., and in these cases, the blood flow abnormalities in the arterial circulation system play a key pathological role. Actually, a large part of mortality rate of adult humans in advanced countries, for example, in Japan, the number of people who died from cerebrovascular and cardiovascular diseases is almost the same as that of people who died from cancers [1].

Usually, the blood-pressure is measured at a brachial region using a cuff by observing Korotkoff sounds. However, this measurement only gives us the peak blood-pressure of ejection wave and that of repulsive wave is not measured. The central blood-pressure, that is, the blood-pressure of repulsive wave is very important to measure the stiffness of blood vessels and some such apparatuses with a noninvasive way have already been found in the market [2]. However, they are far from wearable, cannot be used in round-the-clock way and are also expensive. These days, with the advancements of electrical engineering technology, the IoT and wearable devices measuring the vital signs can be in practical use [3]. The authors think that a wearable round-the-clock central blood-pressure gauge can be fabricated as a hardware, however, the software which can accurately estimate the central blood-pressure noninvasively is the problem to be solved.

The authors have already suggested the application of Model Based (MB) approach and actually developed the cardiovascular simulator and its feasibility is proven against 
typical vital data [4]. This means that the central blood-pressure can be estimated from the radial blood-pressure by using the simulator. Therefore, the central blood-pressure measurement system consisting of a wearable IoT device (that is, a sensor), a network and a cloud sever on which the simulator is executed can be constructed [5]. A real-time and round-the-clock blood-pressure measurement is often required. However, the estimation by the simulator requires around ten minutes just for estimating a central blood-pressure from one cycle of pulse wave.

This paper gives a constitutive solution to this estimation problem, that is, an aorta model in the simulator can be represented by a Pressure Transfer Function (PTF) [5]. Using the PTF, the central blood-pressure calculation becomes simple and it is accelerated to the practical level. This PTF representation problem of aorta has attracted the researchers' attention and in this sense, this paper also gives a numerical solution to this pending problem. Moreover, by using the PTF, a real-time central blood-pressure measurement system consisting of wearable gauges, gateways and a main statin (cloud server) is experimentally developed. The evaluation results show the feasibility of the system.

In the followings, Chapter 2 describes the modeling of cardiovascular system, the co-simulator consisting of constructed models, the Pressure Transfer Function modeling the aorta and the monitoring system. In Chapter 3, the prototype system consisting of hardware and software is shown. Chapter 4 deals with the experimental results using the prototype real-time blood-pressure measurement system. The functions are verified and the performances are evaluated under the actual usage. Finally, in Conclusions, the feasibility of the developed system is summarized.

\section{Cardiovascular Simulator}

The cardiovascular simulator proposed and developed by the authors $[4,6]$ is described in this chapter.

\subsection{Modeling of Cardiovascular System as Electrical Circuit}

When the blood flow is stationary, the arterial system is characterized as an electrical circuit. Here, such a relationship as [blood-pressure $]=$ [total peripheral resistance $] \mathrm{x}$ [cardiac output], that is, Ohm's law, can be obtained [4]. This equation is generally used in the clinical practice. However, the actual blood flow is pulsatile and a dynamic resistive property, called hemodynamic impedance, must be considered. Then, this dynamic feature is represented by the combination of electrical devices. For example, the distensibility or stiffness of blood vessels and the inertia of blood flow are modeled by a capacitor and a coil (inductor), respectively.

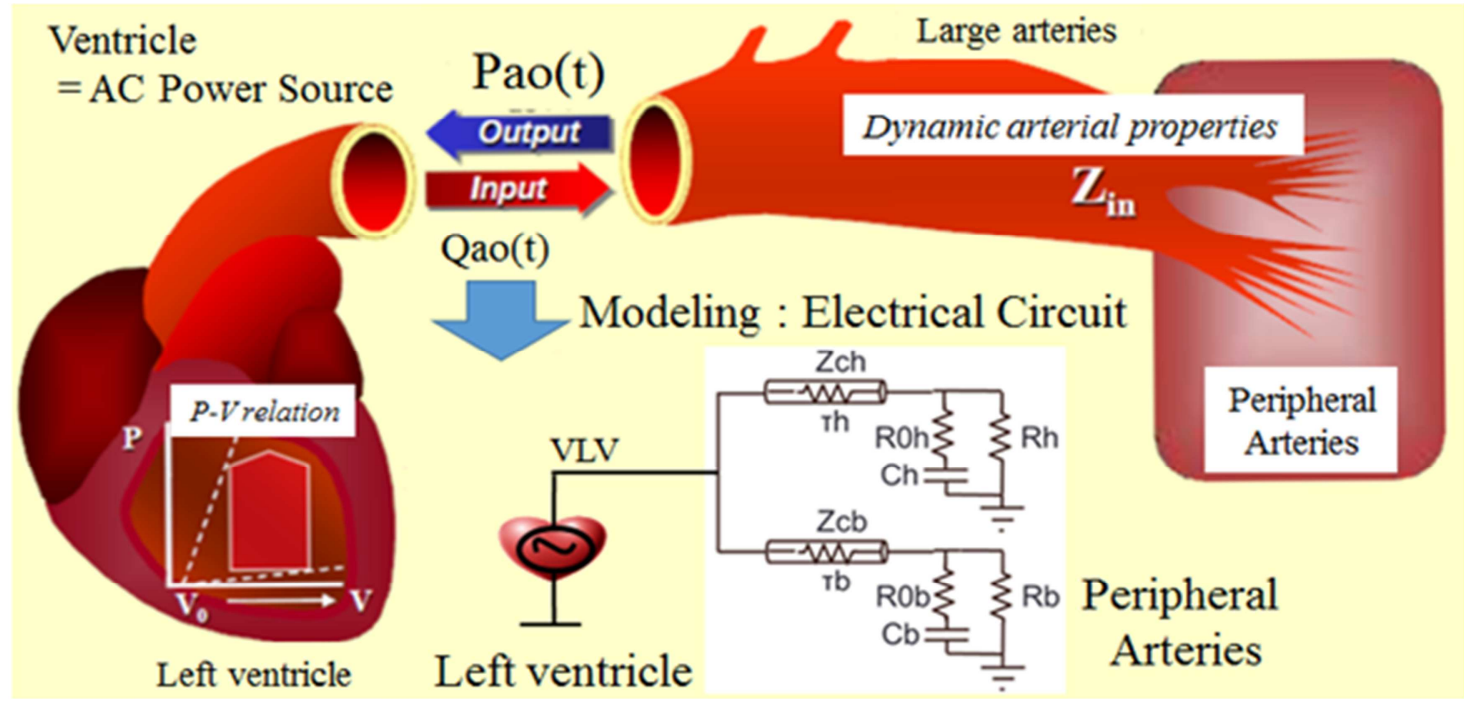

Figure 1. Modeling of Cardiovascular System.

As a result, the whole cardiovascular system is modeled as shown in Figure 1 by using an asymmetric T-tube model for aorta and peripheral arteries [7]. In this figure, a ventricle is modeled as an AC (Alternate Current) power source, the aorta is modeled as a conducting cable with characteristic impedance $\left(Z_{\text {in }}\right)$ and peripheral arteries are modeled as a lumped electrical circuit consisting of parallel resistance and capacitance properties.

\subsection{Arterial System Modeling}

The arterial system is modeled by the circuit whose upper and lower parts correspond to the head and body peripheral arteries, respectively, in Figure 2. This system is an afterload of heart configured by input resistance or characteristic impedance of the aorta, $Z_{\mathrm{ch}}$ or $\mathrm{Z}_{\mathrm{cb}}$. The model is based on the asymmetric T-tube consisting of parallel resistance and capacitance properties [7]. The asymmetrical characteristics are represented by the device parameters in the upper and lower circuits. Here, the peripheral arterial resistances, $\left(R_{0 h}, R_{h}\right)$ and $\left(R_{0 b}, R_{b}\right)$, represent the resistance of blood flow, and the total arterial compliances, $\mathrm{C}_{\mathrm{h}}$ and $\mathrm{C}_{\mathrm{b}}$, indicate the Windkessel function of elastic vessel $[6,8]$. In this figure, the central blood-pressure and the radial 
blood-pressure correspond to the voltages at $V_{c}(t)$ and the voltage at $\mathrm{V}_{\mathrm{r}}(\mathrm{t})$, respectively. By executing the circuit simulation, $V_{c}(t)$, which is now measured by using, for example, a catheter invasively, can be calculated by $\mathrm{V}_{\mathrm{r}}(\mathrm{t})$, which can be measured non-invasively. This is the great advantage of using the cardiovascular simulator.

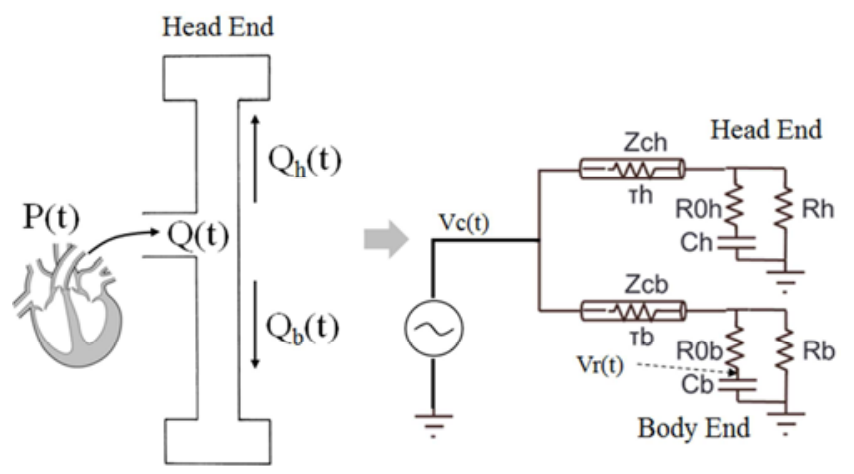

Body End

Figure 2. Asymmetric T-tube Model for Arterial System.

\subsection{Implementation of Suggested Simulator}

The above mentioned model is implemented by MATLAB/Simulink [4, 9] and the necessary parameter values can be simultaneously obtained as the corresponding device parameter values. Actually, as mentioned above, the central blood-pressure can be calculated from the pressure of pulse wave at radius, which is the usual measurement method at clinical use.

The top view of developed simulator is shown in Figure 3. The small four blocks around the upper left corner realize each function of left ventricle, that is, "Q $Q_{\text {in }}$ Calc.", the volume of venous return; "VLV Calc.", the ventricular volume; "PLV Calc.", the ventricular pressure; and " $\mathrm{Q}_{\mathrm{Ao}} \& \mathrm{P}_{\mathrm{Ao}}$ Calc.", the volume of cardiac output. These calculations are based on the Ohm's law [4]. As an example, the inside of the block " $Q_{\text {in }}$ Calc." is partly shown in Figure 4 and the calculation process is described by MATLAB scripts. These scripts are also represented by the combination of simple arithmetic blocks in MATLAB, however, the scripts are easy to understand and fast for execution.

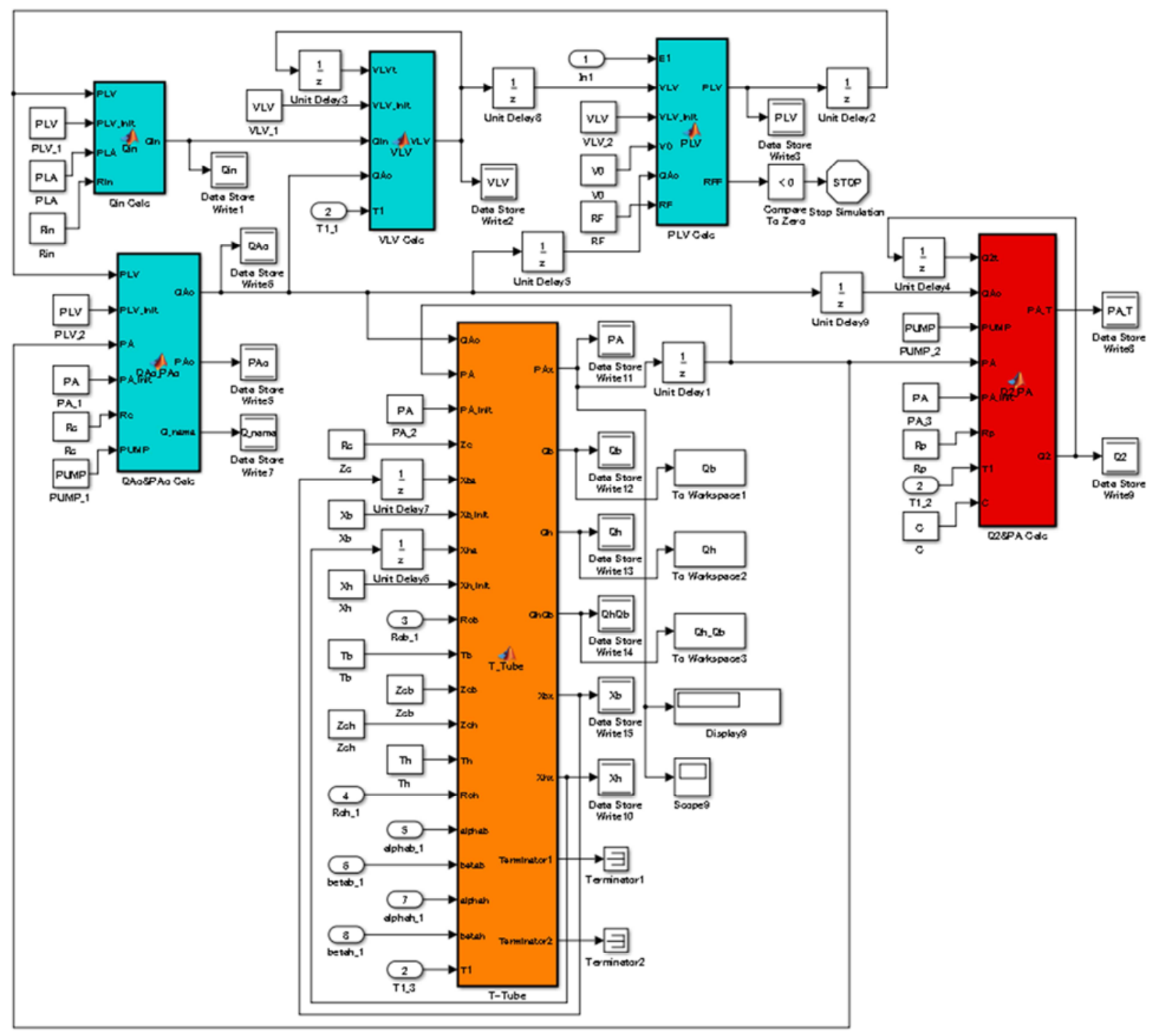

Figure 3. Top view of cardiovascular simulator 
The largest block, "T-Tube", in the bottom of Figure 3 represents the asymmetric T-tube model for arterial system. The lines of MATLAB scripts for the blocks, "Q $Q_{\text {in }}$ Calc.", "VLV Calc.”, "PLV Calc.", "Q Ao $_{\text {\& }} P_{\text {Ao }}$ Calc." and "T-Tube" are 17, 13, 15, 22 and 38, totally, only 105 lines. The descriptions are very simple.

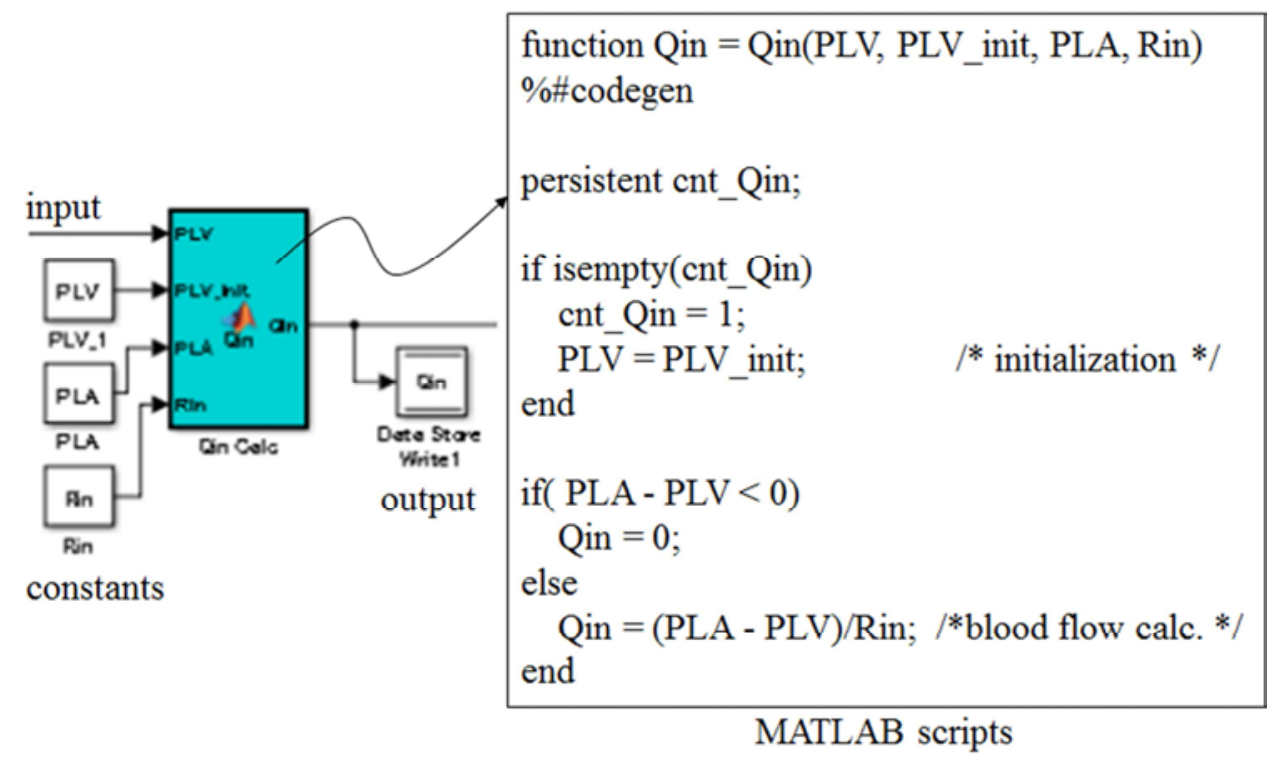

Figure 4. Inside of a Part of " $Q_{\text {in }}$ Calc".

\subsection{Simulation-Driven Parameter Estimation}

Some valuable parameters in cardiovascular system can be estimated by executing the developed simulator. Actually, this simulator makes it possible that the pair of parameters, that is, the maximum ventricular elastance and the peak deviation factor of ventricular elastance which cannot be measured simultaneously can be estimated [4]. Here, the simulation-driven parameter fitting approach is utilized and the number of iterations for the termination is 11 . The used CPU time is 1 hour 43 minutes and 41 seconds. Almost all of the CPU time is consumed for executing the simulator. Therefore, if the simulation-driven parameter fitting is practically used, some kind of acceleration is indispensable.

\subsection{Fast Estimation of Central Blood-Pressure}

The non-invasive central blood-pressure measurement is a very important problem as mentioned above. By using the developed cardiovascular simulator and the simulation-driven parameter fitting, the central blood-pressure estimation is possible from the pulse wave at the radius. Here, the problem is that this parameter fitting needs a huge amount of time.

In order to accelerate the parameter fitting, it is suggested that the aorta model, "T-Tube" block in Figure 3, is replaced by the corresponding transfer function, called a Pressure Transfer Function (PTF) [5]. The transfer function is commonly used in the analysis of systems such as single-input and single-output filters and its calculation is usually very fast.

By using the PTF, the central blood-pressure can be obtained from the observable radial pulse wave pressure as shown in Figure 5. The process is as follows: (i) transform the measured radial pulse wave pressure $p_{2}(t)$ to $P_{2}(f)$ by using Fast Fourier Transform (FFT), (ii) calculate $P_{l}(f)$, which is the FFTed central blood-pressure and (iii) apply inverse FFT (IFFT) to $P_{l}(f)$ to obtain $p_{l}(t)$. This process is very simple because only FFT, division of complex numbers and IFFT at each observation of $p_{2}(t)$ are applied. The acceleration is verified in Chapter 4.

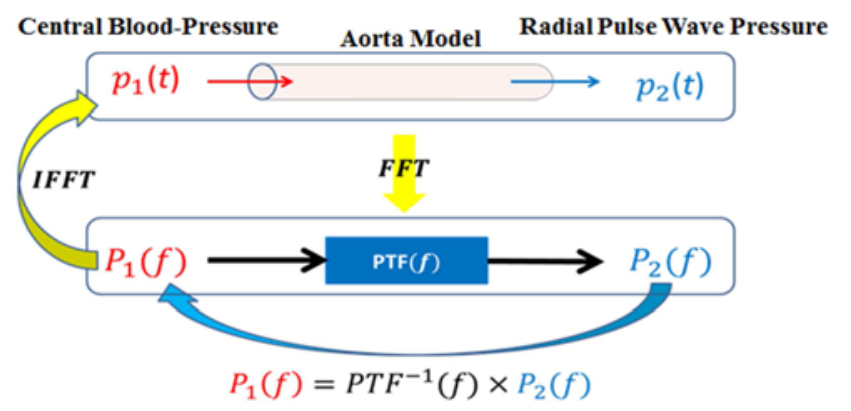

Figure 5. Pressure Transfer Function for Aorta Model.

\subsection{Pressure Transfer Function for Aortal Model}

The Pressure Transfer Function (PTF) for aortal model is numerically obtained by MATLAB scripts. The set of MATLAB scripts is totally 64 lines and its PTF calculation part is shown in Figure 6. The variables $\mathrm{a}$ and $\mathrm{b}$ are the input and the output variables, respectively. These variables are transformed by FFT from time-domain to frequency-domain in the first and second lines. In the next for-loop, these results are transformed into complex numbers. In the second for-loop, the PTF is actually calculated. Then, in the third for-loop, the obtained PTF is verified, and this process is not necessary when only PTF is calculated. The last line changes the domain of central blood-pressure from frequency to time by applying inverse FFT. The obtained numerical set of data is incorporated as a Look Up Table in the simulator replacing "T-Tube" block in Figure 3. 


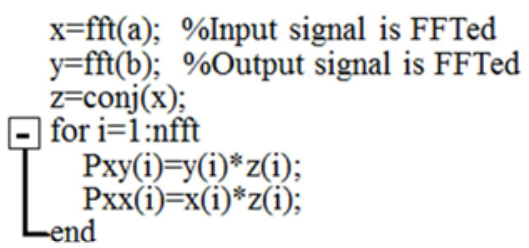

\%Transfer Function Calc. in Frequency Domain
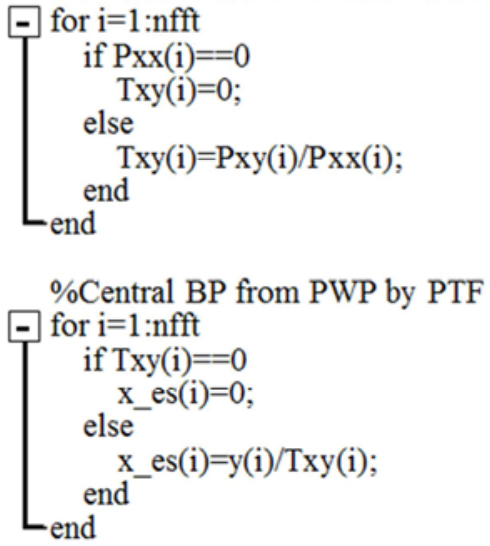

$\mathrm{xx}=\mathrm{ifft}\left(\mathrm{x} \_\mathrm{es}\right) ; \quad \%$ Central BP is inverse FFTed

Figure 6. MATLAB Scripts for Calculating PTF.

\subsection{Data Collecting and Monitoring System}

The round-the-clock observation of central blood-pressure continuously is very important because the blood-pressure varies much with the human activity.

\subsubsection{System Configuration}

The pulse wave collecting and examinee monitoring system is suggested. The system configuration shown in Figure 7 consists of main station (cloud server), gateways and wearable sensors (gouges). The communication between gateways and sensors is based on Bluetooth Low Energy (BLE) in order to reduce the power dissipation. A certain number of gateways are necessary to collect the data from sensors as well as to estimate the locations of sensors (locations of examinees wearing sensors). The protocols between the main station and gateways as well as between gateways and sensors are specifically designed for this system. The central blood-pressure of each examinee is calculated in the main station using the above mentioned simulator and his/her own PTF from the observed pulse wave. Therefore, the necessary parameters and the PTF of each examinee must be saved in the cloud of the main station in advance.

\subsubsection{Localization Method for Sensors}

As a localization of examinees, the Centroid Localization (CL) method is popular. Some variations of CL methods have already been suggested $[10,11]$. In the followings, the CL method is modified in order to estimate the position of sensor. The distance between the gateway and the sensor specified by the protocol is obtained at the main station from the RSSI (Received Signal Strength Indicator) value based on the following equation.

$$
R S S I=-\left(10 n \log _{10} d+A\right)
$$

From this equation, the distance $d$ is obtained as follows.

$$
d=10^{\wedge}(A-R S S I) / 10 n
$$

where, $A$ is an RSSI reading at one meter distance from the sensor, $n$ is the signal transmission constant which is relevant to signal transmission environment and $d$ is the distance from the sensor node to the gateway. The parameters $A$ and $n$ are calibrated in each environment at the first time.

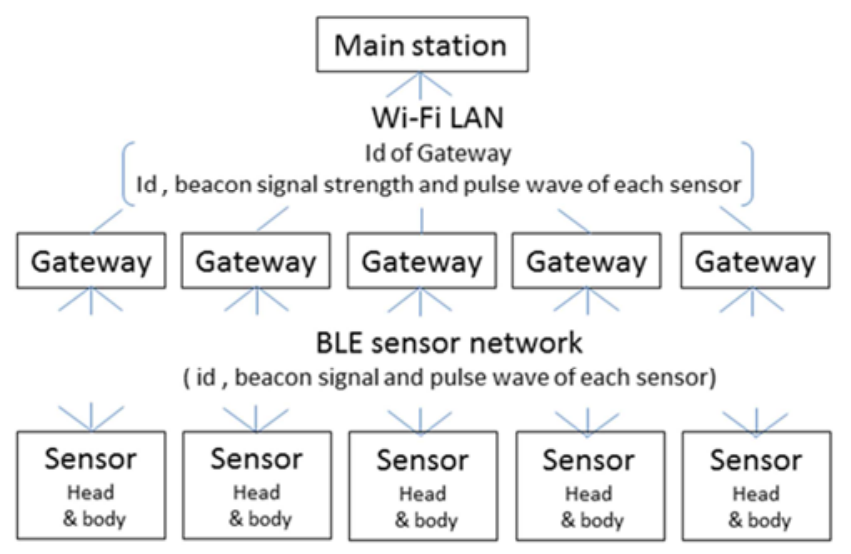

Figure 7. Suggested System Configuration.

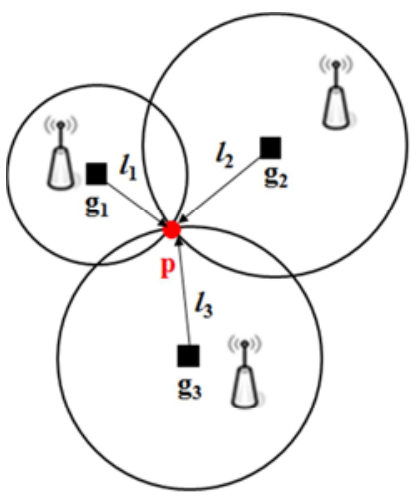

(a) one-point intersection of centroid

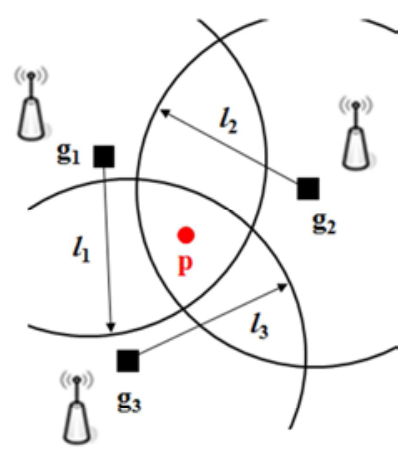

(b) multiple-point intersection of centroid
Figure 8. Localization: Theory VS. Reality.

Ideally, the triangulation gives only one intersection based on the distances as in Figure 8(a). In this case, the positioning has no problem. However, because the signals have significant noise in reality, the triangulation has multiple intersection points as in Figure 8(b). Here, the Least Square Estimation (LSE) method is adopted to approximate the position estimation. The LSE method requires the following equation based on the distance measurement in the previous step. In the followings, the distance between two points in the two dimensional space is represented by the next Euclidian distance.

$$
d\left(p, g_{1}\right)=\sqrt{\left(x-x_{1}\right)^{2}+\left(y-y_{1}\right)^{2}},
$$


where, the coordinates of $p$ and $g_{1}$ are $(x, y)$ and $\left(x_{1}, y_{1}\right)$, respectively. Then, the least square error of estimation of $p$ is calculated by the following equation,

$$
E(p)=\left(l_{1}-d\left(p, g_{1}\right)\right)^{2}+\left(l_{2}-d\left(p, g_{2}\right)\right)^{2}+\left(l_{3}-d\left(p, g_{3}\right)\right)^{2}
$$

Therefore, the LSE method minimizes the $E(p)$ by changing the coordinate $(x, y)$ of $p$.

\section{Prototype of Central Blood-Pressure Measurement System}

\subsection{Main Station}

The main station collects the pulse wave data and the data for localization of sensors. It is designed that around forty examinees are simultaneously monitored in the system. The processes on the main station are to obtain the central blood-pressure from the pulse wave data and to estimate the positions of sensors. All the necessary parameters and the Look Up Table of PTF of each examinee must be accessed from the main station. This main station has an Intel Core i5 CPU, 2.4GHz clock frequency and 4GB main memory. If the operator wants to check the examinee's pulse wave, central blood-pressure and the location, these data can be shown on the main station by selecting the examinee's ID in the pull-down menu. The actual snapshot is shown in Chapter 4.

\subsection{Gateway}

The gateway collects the data detected by each of the wearable gauges (sensors). The data are transmitted to the main station. The gateway is implemented by using a Raspberry Pi model 3 [12]. A micro SD card is installed for the data backup as shown in Figure 9.

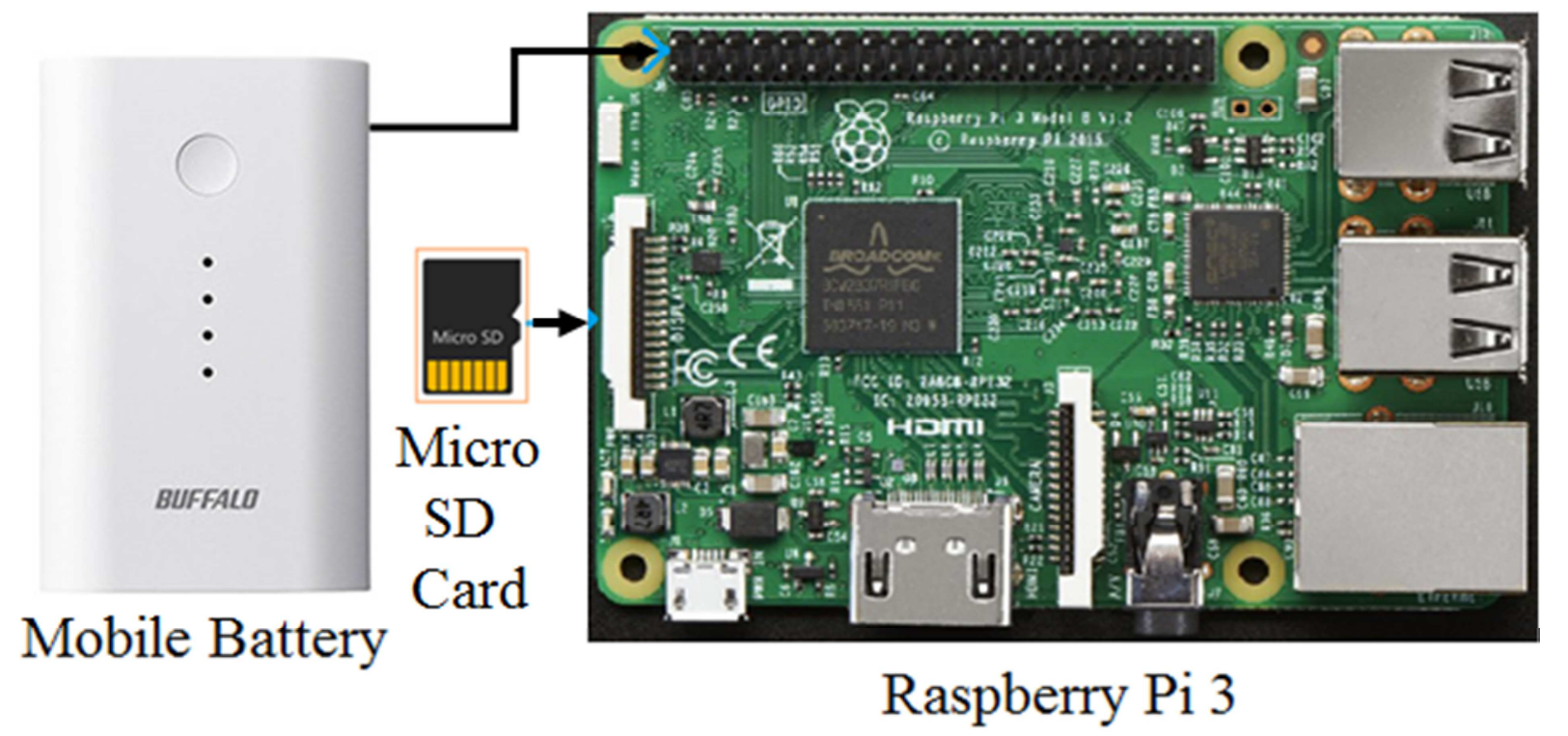

Figure 9. Gateway Implementation.

\subsection{Wearable Gauge}

The wearable gauge is implemented by using BLE (Bluetooth Low Energy) microcomputer [13] for reducing the battery usage as shown in Figure 10. Its size is currently not enough small as a wearable gauge but the size can be reduced when it is implemented by an Application Specific Integrated Circuit. Here, at least now, not a radial pulse wave pressure but a digital pulse volume is measured because no compact and cost-effective sensors measuring the radial pulse wave pressure are available. Therefore, the digital pulse volume must be transformed to the radial pulse wave pressure before applying the PTF and this transformation is now being developed.

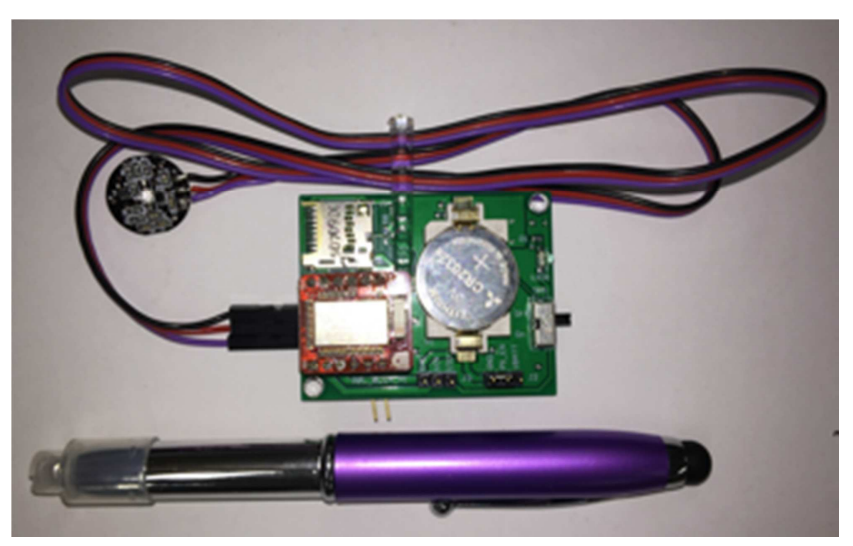

Figure 10. Wearable Gauge (37mm x 50mm). 


\section{Experimental Results}

The feasibility of suggested central blood-pressure measurement system with the wearable gauge is evaluated. The functions of the system are firstly checked and then, the performances are evaluated.

\subsection{Accuracy of Pressure Transfer Function}

The accuracy of Pressure Transfer Function (PTF) which is expected to replace the aorta model is evaluated against three data types of human cardiovascular systems [14]. For all data types, the mean square errors (MSE) between the central-blood pressures of simulator and those of PTF are 2.0e-14, 2.4e-14, and 4.1e-14, and therefore, the accuracy is within a practical use [5]. The details of the data types are described [5]. The central blood-pressures obtained from PTF and the simulator are shown in Figure 11. The $\mathrm{x}$ and $\mathrm{y}$ axes are the time (seconds) and the blood-pressure $(\mathrm{mmHg})$, respectively. Two waveforms cannot be distinguished visually because its MSE is 4.1e-14. Of course, these waveforms represent the characteristics of central blood-pressures for young adulthoods [14]. The CPU time of PTF-based approach is less than a second.

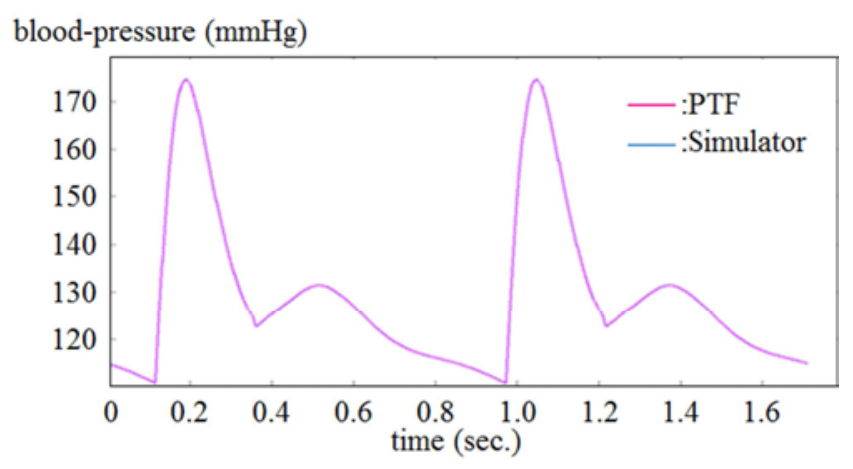

Figure 11. Central Blood-Pressure Waveforms Obtained by PTF and by Simulator (Young Adulthood).

\subsection{Estimation of Central Blood-Pressure}

A snapshot of the developed central blood-pressure measurement system is shown in Figure 12. The current system consists of four wearable gauges and five gateways. An arbitrary examinee's measurement data, that is, digital pulse volume and heart rate can be displayed as in the figure. The upper part is the waveform of actual digital pulse volume and this is refreshed every second. Then, an examiner can select an arbitrary part from the waveform of digital pulse volume and the selected waveform, shown in the bottom left, is used for central blood-pressure estimation. The estimated waveform of central blood-pressure is shown in the bottom right less than a second.

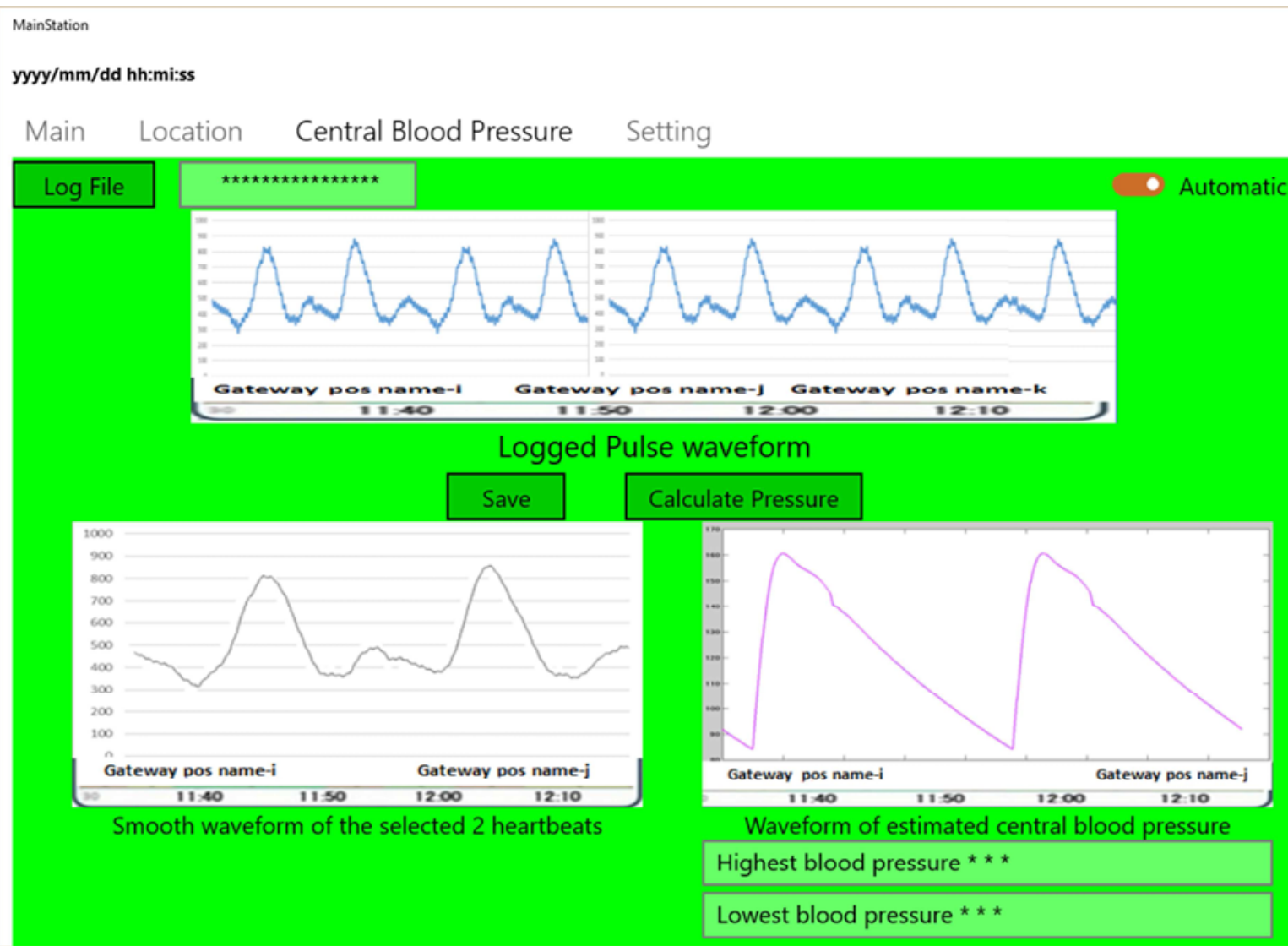

Figure 12. Snapshot of Developed Measurement System on Main Station. 


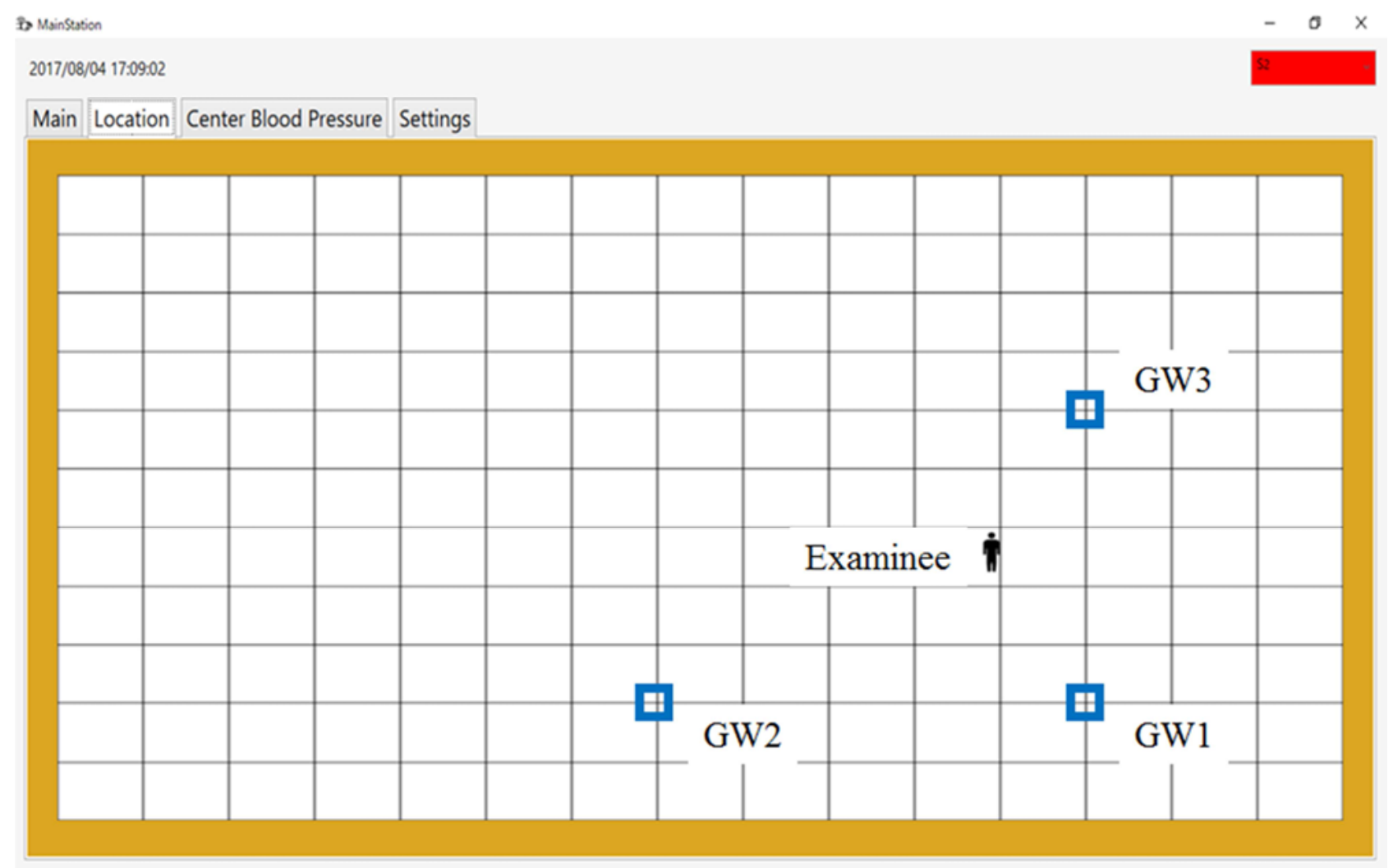

Figure 13. Snapshot of Examinee'S Location Display on Main Station.

\subsection{Evaluation of Centroid Localization Method}

The room $(15 \mathrm{~m} \times 11 \mathrm{~m})$ shown in Figure 13, which corresponds to one floor of care facility, hospital, or health care center, etc., is used for the evaluation. Here, the expression " $d=10^{\wedge}(A-R S S I) / 10 n$ " described in 2.7.2 is used with $\mathrm{n}=2.0$ meaning an ideal area with no obstacles. In Figure 13 , the locations of three gateways and the location of examinee wearing a gouge are shown with labels, GW1, GW2, and GW3 and Examinee, respectively.

In the experiment, the errors between the actual and

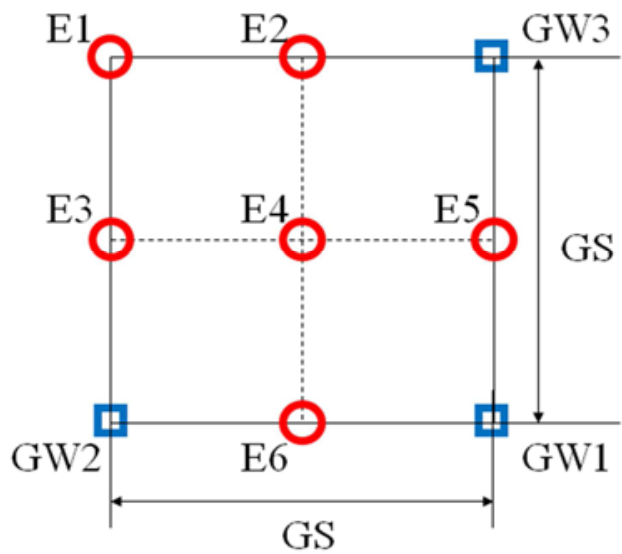

(a) Locations of examinee estimated locations of examinee are evaluated with the varying gateway separations (GS), of (GW1, GW2) and (GW1, GW3) by $1 \mathrm{~m}, 2 \mathrm{~m}, 3 \mathrm{~m}, 4 \mathrm{~m}$, and $5 \mathrm{~m}$. The locations of examinee are set on each of grid points E1, E2, E3, E4, E5, or E6, as shown in Figure 14(a). The evaluation is to check the errors (meters) between the actual and the estimated locations of examinee. That is, when the examinee is supposed to be at E1 and gateway separation is $1 \mathrm{~m}$, it is evaluated how distant the estimated location is from E1.

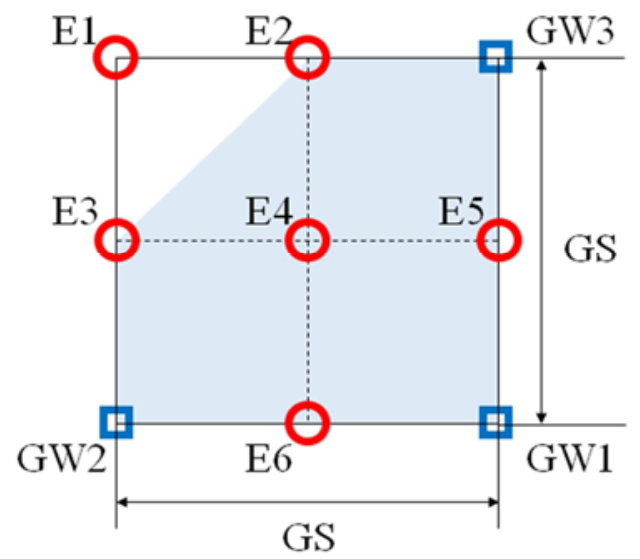

(b) Area with practically small location errors

Figure 14. Locations of Gateways and Examinee and Area with Practically Small Location Errors.

Table 1 shows the results with the variations of gateway separations and examinee's locations. In this table, for example, when the gateway separation is $1 \mathrm{~m}$ and the examinee is at E1, it is shown that the estimation error is 5.0 
m. The evaluation results in Table 1 indicate that the average estimation error is less than $2.5 \mathrm{~m}$ when the gateway separation is less than $3 \mathrm{~m}$ and the examinee locations are E2, E3, E4, E5, and E6 as shown by the shaded cells in the "Ave." and "Std." columns. This corresponds to the shaded area in Figure 14(b). This means that if examinees are in this area, their locations can be estimated within $2.5 \mathrm{~m}$ errors when GS is less than $3 \mathrm{~m}$. Some papers (e.g. [15]) report the accuracy improvement of positioning based on BLE and the estimation error is maximally $1.7 \mathrm{~m}$ or $2.48 \mathrm{~m}$ when a cell phone is used as a transmitter or a receiver. In the use of the developed system, the objective of localization is to know whether an examinee is moving or stopping or he/she is in the specified room or not. Therefore, the suggested centroid localization method can be used for this system. The accuracy of localization would be improved by optimizing the gateway placements or replacing BLE by Zigbee, Bluetooth or Wi-Fi.

Table 1. Errors of Variations of Gateway Distance and Examinee'S Locations.

\begin{tabular}{llllllll}
\hline \multirow{2}{*}{$\begin{array}{l}\text { Location of } \\
\text { Examinee }\end{array}$} & \multicolumn{7}{c}{ Gateway Distance $(\mathbf{m})$} \\
\cline { 2 - 8 } & $\mathbf{1}$ & $\mathbf{2}$ & $\mathbf{3}$ & Ave. & Std. & $\mathbf{4}$ & $\mathbf{5}$ \\
\hline E1 & 5.0 & 1.4 & 5.1 & 3.8 & 1.7 & 7.8 & N/A \\
E2 & 0.5 & 1.0 & 1.5 & 1.0 & 0.4 & 6.0 & 7.8 \\
E3 & 2.0 & 4.1 & 1.5 & 2.5 & 1.1 & 2.0 & N/A \\
E4 & 0.0 & 3.2 & 4.1 & 2.4 & 1.8 & 4.1 & 5.0 \\
E5 & 2.1 & 0.5 & 2.5 & 1.7 & 0.9 & 2.5 & 1.0 \\
E6 & 0.5 & 1.0 & 1.5 & 1.0 & 0.4 & 9.8 & 3.0 \\
Ave. & 1.7 & 1.9 & 2.7 & - & - & 5.4 & N/A \\
\hline
\end{tabular}

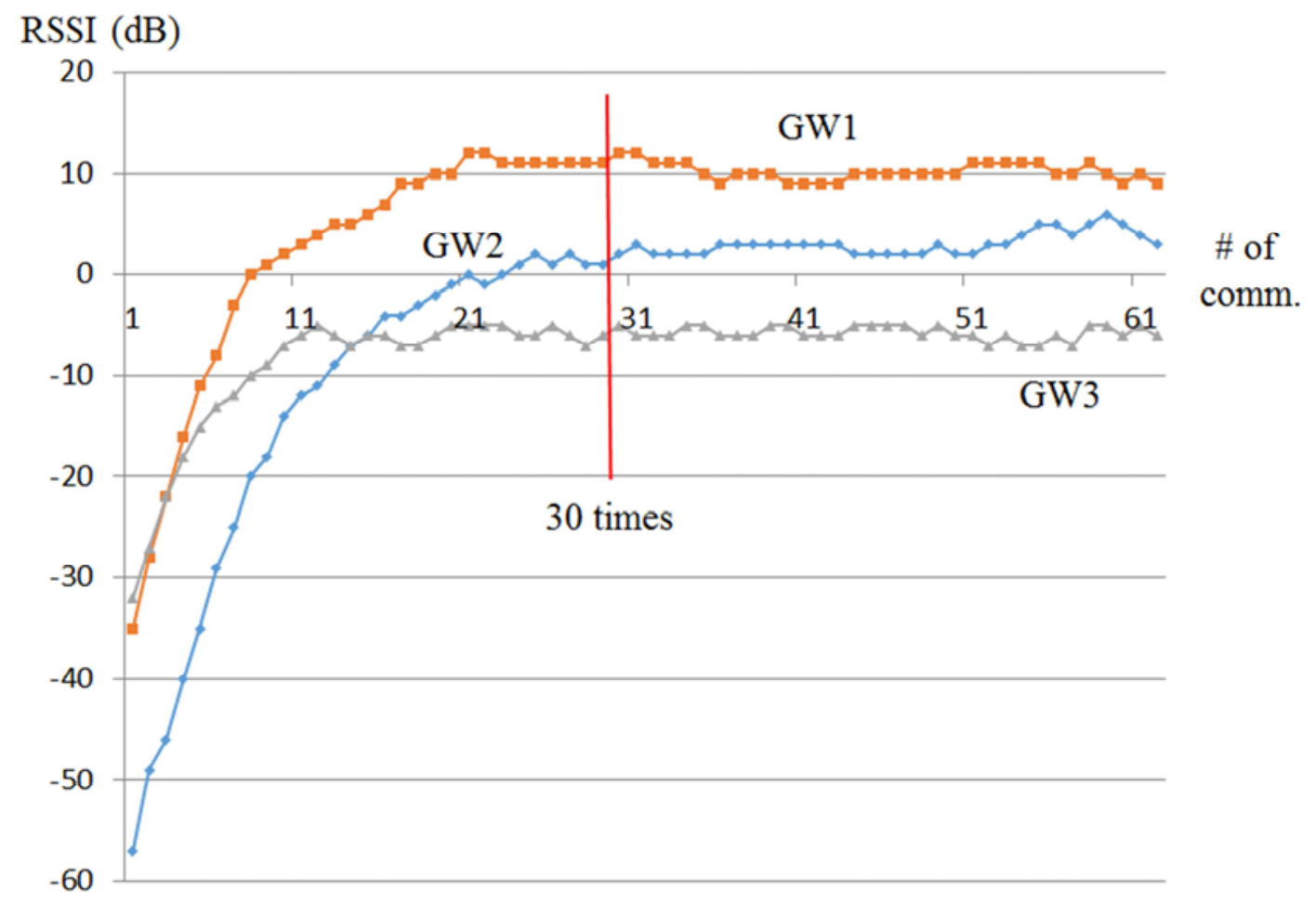

Figure 15. RSSI Values VS. Number of Communications.

Figure 15 shows the relationship between the RSSI values and the number of communications after the pairing. Although the pairing is automatically done, it should be noted the RSSI values are unstable less than 30 times of communications when an unpaired gauge is newly added in the location estimation system. The cycle time of communications of BLE ranges from 7.5 milliseconds to 4 seconds depending on the device and therefore, the corresponding warming-up period (maximally 120 seconds) is needed before this system is available only when a new gauge is added.

In all of these experiments, the digital pulse volume data are normally received to the main station. The wearable gauge can work about six hours equipped with the coin battery shown in Figure 10 in such a usage as in these experiments. In order to prolong the operating time of gauge and to replace the BLE with Zigbee or Bluetooth for improving the localization accuracy, an energy harvesting technology or some other energy saving methods are considered effective.

\section{Conclusions}

A wearable central blood-pressure measurement system is proposed, developed and its performances are evaluated. This system consists of noninvasive wearable gauges, set of gateways relaying the measurement data and a main statin (cloud sever) estimating the location of examinees wearing the gauge as well as calculating the central blood-pressure. Based on the evaluation results, the proposed system is likely to be used practically for measuring the central blood-pressure and for estimating the location of examinee in a real-time way for certain period of time. As future work, the accuracy of central blood-pressure estimation should be evaluated by precisely measuring the radial pulse wave pressure instead of the digital pulse volume. Moreover, some 
energy harvesting technology should be incorporated for implementing a round-the-clock wearable gauge.

\section{Acknowledgements}

The authors express their sincere appreciation to Mr. Le Ngoc Khani, Mr. Yoshinori Hatano and Mr. Tamio Hoshino in Applistar Corporation and to Mr. Yasuhiro Kobayashi in Realize Computer Engineering for developing the prototype measurement system. This research was funded by the Ministry of Economy, Trade and Industry, Japan.

\section{References}

[1] Vital Statistics in JAPAN -The latest trends- Website, http://www.mhlw.go.jp/english/database/db-hw/d1/81-1a2en.p df.

[2] Automatic blood pressure monitor / arm / with central aortic pressure monitor $\mathrm{BP}+$, Website http://www.medicalexpo.com/prod/uscom/product-70427-543 975.html.

[3] Biosignalplux, wearable body sensing platform, Website, http://biosignalsplux.com/index.php/en/.

[4] S. Takahashi, K. Sakawa, Y. Shiraishi and H. Miyashita, "Modeling, Simulation and Parameter Estimation of the Cardiovascular System by using Model Based Approach," SICE 2013 Annual Conference, pp.493-500, Hokkaido, September, 2013.

[5] K. Nakajo, Y. Komori, S. Takahashi, K. Motegi, Y. Shiraishi, and H. Miyashita, "Pressure Transfer Function for Aorta Model in Cardiovascular Simulator, Feasibility Study of Wearable Central Blood-Pressure Gauge," International Conference on Medical Engineering, Health Informatics and Technology (MediTec 2016), Dhaka, December 17-18, 2016.

[6] H. Miyashita, M. Sugimachi, T. Sato, T. Kawada, T. Shishido, T.
Nakahara, R. Yoshimura, H. Takaki, H. Miyao, and K. Sunagawa, "A novel servo-control system that imposes desired aortic input impedance on situ rat heart," Am. J. Physiol. Heart Circ. Physiol. 278: H998-H1007, 2000.

[7] K. B. Campbell, R. Burattini, D. L. Bell, R. D. Kirkpatrick and G. G. Knowlen, "Time-domain formulation of asymmetric T-tube model of arterial system," Am. J. Physiol. Heart Circ. Physiol. 258:H1761-H1774, 1990.

[8] W. W. Nichols, M. F. O'Rourke, C. Vlachopoulos, "McDonald's Blood Flow in Arteries," Theoretical, Experimental and Clinical Principles, 6th ed. London: Hodders Arnold, 2011.

[9] Math Works, Website, http://www.mathworks.co.jp/products/pfo/.

[10] N. Bulusu, J. Heidemann, and D. Estrin, "GPS-less low cost outdoor localization for very small devices," IEEE Personal Communications Magazine, Vo.7, No.5, pp.28-34, October 2000 .

[11] J. Blumenthal, R. Grossmann, F. Golatowski, and D. Timmermann, "Weighted Centroid Localization in Zigbee-based Sensor Networks," IEEE International Symposium on Intelligent Signal Processing, October, 2007.

[12] "RASPBERRY PI3 MODEL B," https://www.raspberrypi.org/products/raspberry-pi-3-model-b/.

[13] “BLE Nano," http://redbearlab.com/blenano/.

[14] J. P. Murgo, N. Westerhof, J. P. Giolma and S. A. Altobelli, "Aortic input impedance in normal man: relationship to pressure waveforms," Circulation 62: pp.105-116, 1980.

[15] N. Guan, Z. G. Wen and K. G. Sun, "Research on Indoor Positioning Algorithm Based on Trilateral Positioning and Taylor Series Expansion," Proceedings of 2016 International Conference on Computational Modeling, Simulation and Applied Mathematics (CMSAM 2016), 3266, Bangkok, July 2016. 